Rüdiger Wilhelmi

\title{
Risikoschutz durch Privatrecht
}

\author{
Eine Untersuchung zur negatorischen und deliktischen Haftung unter besonderer \\ Berücksichtigung von Umweltschäden
}

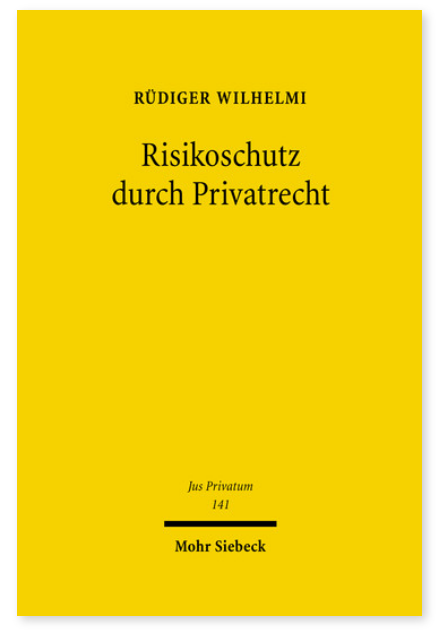

2009. XVII, 430 Seiten. JusPriv 141

ISBN 978-3-16-151218-6

DOI 10.1628/978-3-16-151218-6

eBook PDF $114,00 €$

ISBN 978-3-16-149746-9

Leinen $114,00 €$
Menschliches Verhalten ist regelmäßig mit Risiken für andere verbunden. Der damit notwendigen Abgrenzung der Freiheitsund Risikosphären des Handelnden und der durch dessen Verhalten Betroffenen dienen insbesondere die negatorische und die deliktische Haftung, indem sie den Betroffenen mehr oder weniger Risikoschutz gewähren. Sie stehen dabei in einem funktionellen Ergänzungsverhältnis; während die negatorische Haftung auf Unterlassung und Beseitigung drohender Beeinträchtigungen gerichtet ist, hat die deliktische Haftung den Ersatz des Schadens durch in der Vergangenheit liegende Beeinträchtigungen zum Gegenstand. Rüdiger Wilhelmi untersucht die Voraussetzungen der negatorischen und deliktischen Haftung, die es erlauben, das Haftungsrecht als Schutz der individuellen Freiheit durch subjektive Rechte aufzufassen und trotzdem Allgemeininteressen, etwa am Umweltschutz, hinreichend zu berücksichtigen. Er arbeitet insbesondere heraus, dass neben der deliktischen auch die negatorische Haftung eine Verkehrspflichtverletzung voraussetzt, und bestimmt die Kriterien, die bei der Interessenabwägung zur Ermittlung der jeweils relevanten Verkehrspflichten zu berücksichtigen sind; dabei zeigt er anhand der gesetzlichen Wertungen auf, dass das von der deliktischen Haftung vorausgesetzte Verschulden anders als die Verkehrspflichten subjektiv zu bestimmen ist. Bei der Festlegung der Kriterien misst der Autor dem Risiko, das Erfordernis der negatorischen Haftung, Zurechnungsgrund der deliktischen Haftung und auch zentraler Gesichtspunkt der Verkehrspflichten ist, besondere Bedeutung bei.

Rüdiger Wilhelmi Geboren 1968; Studium der Rechtswissenschaft in Tübingen und Leiden (NL); 2001 Promotion; 2007 Habilitation; Privatdozent für Bürgerliches Recht, Handels- und Gesellschaftsrecht, Wirtschaftrecht und Rechtsvergleichung an der Universität Tübingen.
Jetzt bestellen:

https://mohrsiebeck.com/buch/risikoschutz-durch-privatrecht-9783161512186?no_cache=1

order@mohrsiebeck.com

Telefon: +49 (0)7071-923-17

Telefax: +49(0)7071-51104 